\title{
Investigation of the behaviour of UWB protection elements against HPM signals
}

\author{
F. Brauer, R. Krzikalla, and J. L. ter Haseborg \\ Institute of Measurement Technology and EMC, Hamburg, Germany
}

\begin{abstract}
The development of protection circuits against a variety of electromagnetic disturbances is important to assure the immunity to interferences of an electronic system. In this paper protection elements which were investigated under UWB conditions are now to be tested with HPM signals to achieve a comprehensive protection.
\end{abstract}

\section{Introduction}

Extreme electromagnetic disturbances, like for example ultra wideband (UWB) pulses with rise times below one nanosecond and amplitudes of some kilovolts, represent danger for electronic systems. In addition there are high-energy microwave signals (HPM) with very narrow-band spectra, whereby the centre frequencies lie in the $\mathrm{GHz}$ range.

To guarantee a reliable operation of electronic systems also under these difficult electromagnetic conditions new adapted protection concepts are necessary. In the past protective circuits with nonlinear protection elements have been successfully tested against transient interfering pulses, whose rise times do not fall below the nanosecond range (e.g. LEMP, NEMP and ESD). In preceding investigations different linear and nonlinear filters and protection elements were tested and evaluated for the protection against injected UWB pulses. In particular very fast nonlinear protection elements with extremely small parasitic capacities showed promising results. In this investigation the behaviour is to be tested and evaluated by UWB protection elements regarding HPM signals for the development of a comprehensive system protection against extreme electromagnetic disturbances. It is examined, in which way the tested nonlinear protection elements cause a power limitation of the disturbance and whether this effect is of nonlinear or only linear origin. Finally it is discussed whether the tested protection elements can offer a total protection for electronic systems against UWB and HPM disturbances.

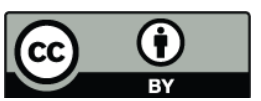

Correspondence to: F. Brauer (f.brauer@tuhh.de)

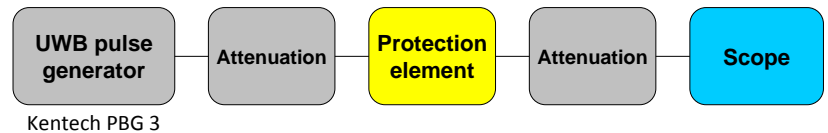

Fig. 1. UWB-measurement setup in time-domain.

\section{Protection devices under UWB-conditions}

In previous investigations different protective circuits (e.g. spark gaps, varistors and protection diodes) have been examined under UWB conditions. For the line-bound UWB pulse generation the pulse generator Kentech PGB3 has been used, which is shown in the schematic measurement setup in Fig. 1. The amplitude of the pulse at the entrance of the respective protective circuit can be varied by the use of attenuation elements. The results of the measurements in the time domain for the slow protection diode BZG03 (left) and the fast protection diode GBLC05 (right) in case of two different amplitudes (1 V and 100 V) are shown in Fig. 2.

It is obvious that the slow protection diode exhibits only a linear response, since pulses of different amplitudes are absorbed in the same way. This behaviour is due to the filter characteristic of the parasitic elements of the diode. Varistors show a similar behaviour, since these elements exhibit relatively high parasitic capacities. Therefore these elements are also not suitable for the protection of RF-signal lines. However the fast protection diode GBLC05 shows a nonlinear response and absorbs a particular amount of the UWB pulse energy. These SMD protection diodes are therefore suitable for the protection of HF-signal-lines against UWB pulses.

\section{Protection devices under HPM-conditions}

In the following HPM pulses are regarded as disturbance signal. These signals represent almost sinusoidal, high frequency oscillation pulses, which can reach field-bound powers of more than $100 \mathrm{MW}$. The frequency of the oscillation is thereby within the range of 0.6 to $10 \mathrm{GHz}$. Pulse widths of $20 \mathrm{~ns}$ to $1 \mu \mathrm{s}$ and pulse repeating rates up to $1 \mathrm{kHz}$ are

Published by Copernicus Publications on behalf of the URSI Landesausschuss in der Bundesrepublik Deutschland e.V. 

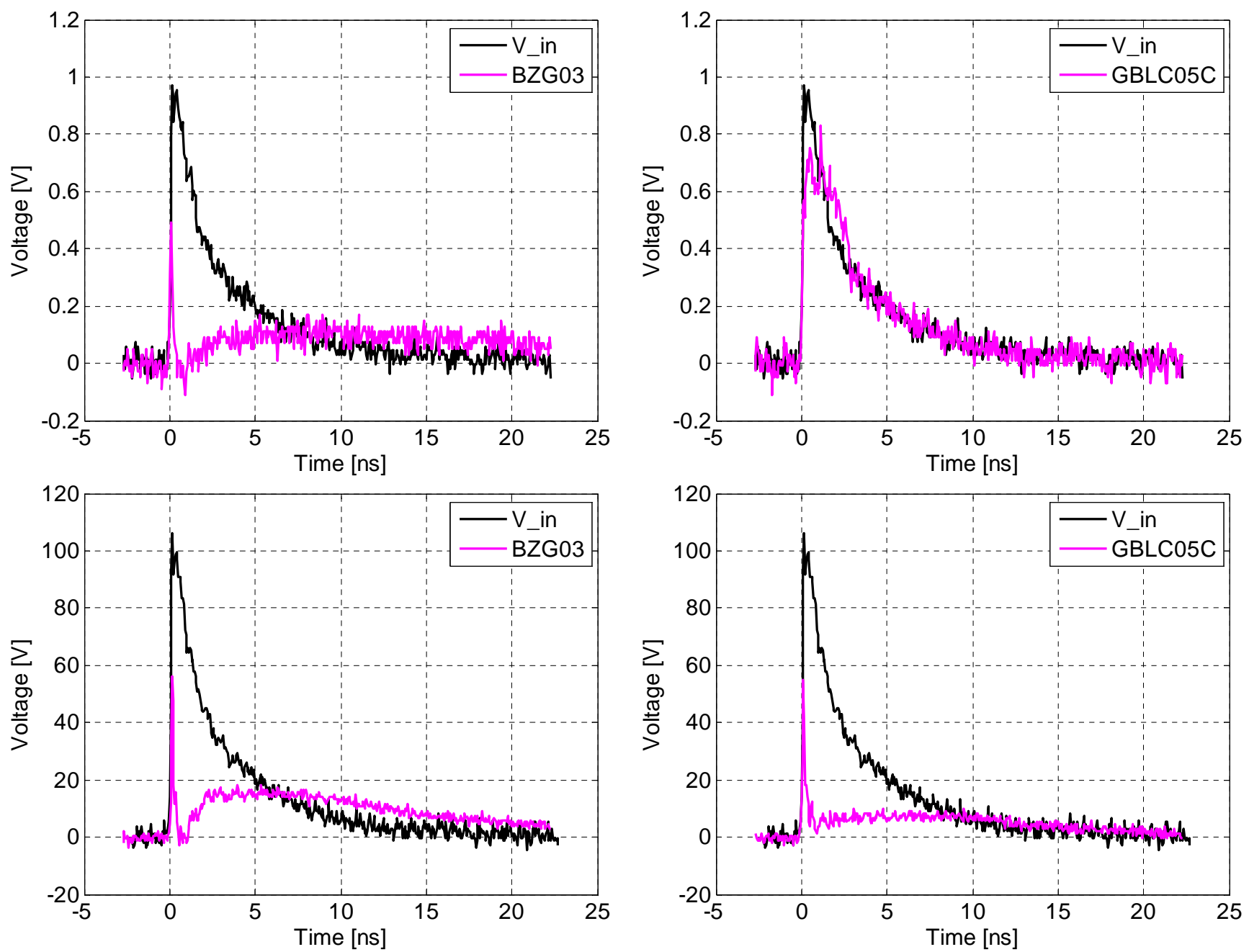

Fig. 2. UWB measurement results.

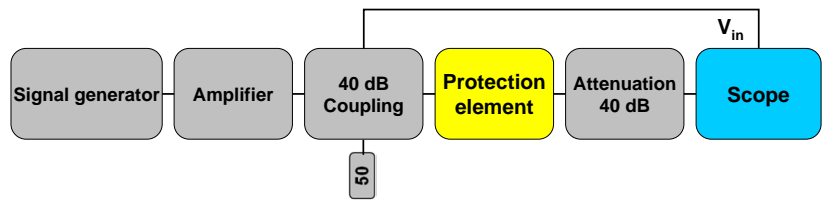

3.1 Measurements in the time-domain

Figure 3 shows the schematic measurement setup for the measurement in the time domain. The protective circuit which is to be examined is stressed with the amplified CW signal of the signal generator. Figure 4 shows the results of the measurements for the diode BZG03 on the left and for the diode GBLC05 on the right at frequencies of $10 \mathrm{MHz}$ and $1 \mathrm{GHz}$ and with different amplitudes of the $\mathrm{CW}$ signal.

reached at the moment. For a first investigation of the protection elements line-bounded continuous wave (CW-) signals up to a power of $50 \mathrm{~W}$ are used in this investigation. Since the art of response and not the thermal behaviour of the protection elements are regarded and with consideration of linear injection behaviour of complex electronic systems, this simplified measuring method can be used here.

In the time domain the nonlinear, unidirectional and/or bi-directional responding of the diodes at a frequency of $10 \mathrm{MHz}$ can be recognized, whereby the fast protection diode responds only with higher amplitudes. At $1 \mathrm{GHz}$ the absorption behaviour is purely linear for both diodes, which is due to the parasitic capacity of the diodes. The phase shift between $\mathrm{V}_{\text {in }}$ and the response signal shows that the parasitic effects especially for the slow protection diode are to be taken into account. 

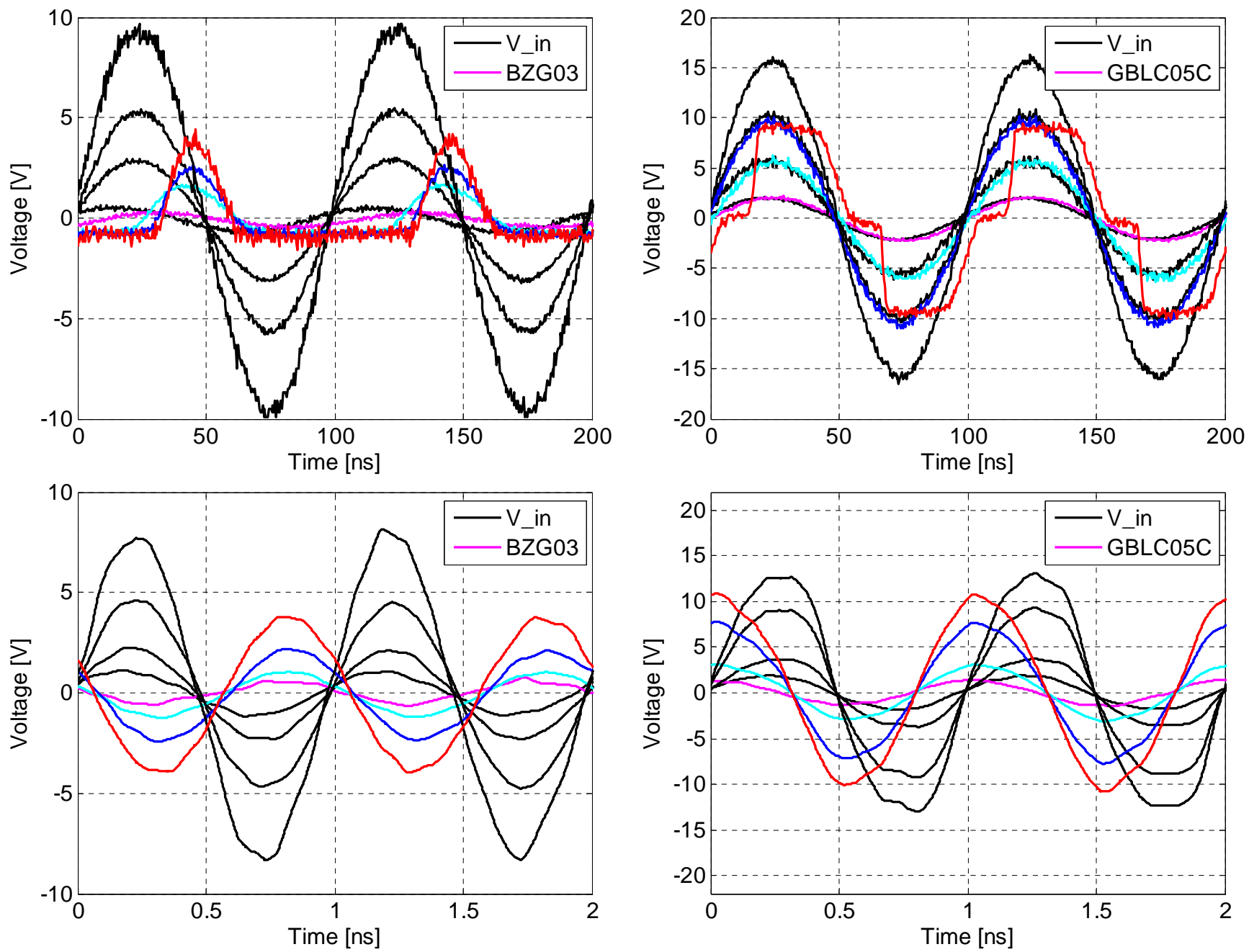

Fig. 4. CW measurement results in time-domain.
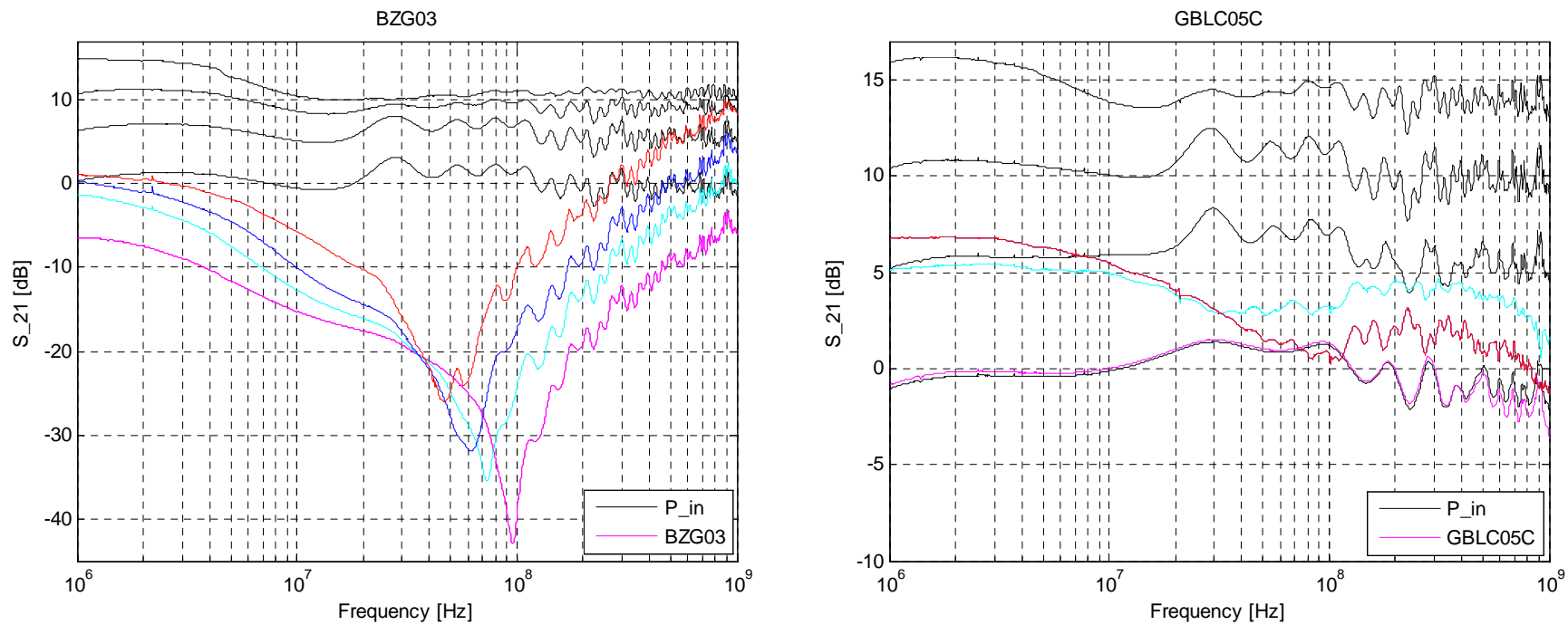

Fig. 5. CW measurement results in frequency-domain. 


\subsection{Measurements in the frequency-domain}

For a better analysis measurements in the frequency domain have been done additionally. A S21-parameter measurement with a network analyzer is used to determine the input power and the transmitted power over a particular frequency range. This range is specified from $1 \mathrm{MHz}$ to $1 \mathrm{GHz}$, since the diodes exhibit no nonlinear responses from a frequency of $1 \mathrm{GHz}$ and higher, as delivered in the time-domain measurements. An investigation of the nonlinear behaviour in the frequency domain is difficult. So the protection diodes are regarded only as power limiters in this case. The results of these measurements are shown in Fig. 5.

The behaviour of the slow protection diode reflects the results of the time domain measurement. This diode is useful up to a maximum frequency of approx. $6 \mathrm{MHz}$. At a frequency of $1 \mathrm{GHz}$ the diode shows approximately no absorption behaviour. The fast protection diode is applicable up to a frequency of approx. $800 \mathrm{MHz}$. Only in case of high signal amplitudes the diode shows a good absorption behaviour.

\section{Conclusion}

These and other results of measurements with further protection elements, which are not presented here, show that UWB protection elements as regarded so far are not suitable for hardening against HPM pulses. For frequencies above $800 \mathrm{MHz}$ the protection elements only react in a linear way. The parasitic effects and the limited response time impair the protective effect of these elements. In future work alternative protection concepts are to be developed and validated by SPICE simulations to ensure the desired protection against any form of HPEM disturbances.

\section{References}

Weber, T., Krzikalla R., and ter Haseborg, J. L.: Linear and Nonlinear Filters Suppressing UWB Pulses, IEEE Transactions on EMC, Vol. 46, No. 3, August, 2004.

Krzikalla, R., Weber, T., and ter Haseborg, J. L.: EMP und UWB Schutz von elektronischen Bauelementen - Final report of the BWB-contract E/E590/1X038/Y5140, October, 2004.

Weber, T.: Messverfahren und Schutzmaßnahmen für elektromagnetische Pulse im UWB-Bereich, PhD Thesis, Hamburg University of Technology, 2004.

Borgeest, K.: Optimierung und Simulation des transienten Ansprech- und Übertragungsverhaltens nichtlinearer Schutzschaltungen für HF-Systeme, $\mathrm{PhD}$ Thesis, Hamburg University of Technology, 1998. 